\title{
Theoretical Interpretation of Magnetic Activity
}

\author{
By EUGENE N. PARKER
}

\begin{abstract}
Enrico Fermi Institute and Departments of Physics and Astronomy, University of Chicago, Chicago, Illinois 60637, USA
\end{abstract}

Magnetic fields generated and driven by thermal convection are the primary cause of solar activity. There are many facets of the activity, such as plages, flares, sunspots, coronal heating, and the variation of solar luminosity or irradiance whose nature and cause are understood only partially or not at all, although detailed superficial observational descriptions are available. It is suggested that the inferred $10^{5}$ gauss azimuthal field bundles may be a direct result of the emergence of $\Omega$-loops to form bipolar magnetic regions on the surface in association with an increase in solar irradiance.

\section{Solar activity}

The surface of the Sun exhibits a variety of suprathermal phenomena collectively called activity. Close examination shows that the activity is mostly magnetic in character, driven by the interaction of convection with magnetic fields that are generated somewhere beneath the visible surface. It is evident, then, that the theory of magnetic activity begins with the perplexing problem of the solar dynamo, generating azimuthal magnetic field with a 22 year periodicity somewhere in or below the convective zone.

The magnetic suprathermal effects observed at the surface of the Sun include faculae, plages, prominences, coronal mass ejections, and flares of all sizes. Then there is the subthermal magnetic sunspot to give variety. The chromosphere, and particularly the corona, are the outward manifestations of the activity. In regions where the mean magnetic field is relatively weak $(<10$ gauss) the coronal gas is heated to a temperature of $1-1.5 \times 10^{6} \mathrm{~K}$ and continually expands outward into space, gradually accelerating to hundreds of $\mathrm{km} / \mathrm{sec}$ to produce the solar wind. The expansion carries the weak field along with it, sweeping out through the solar system to produce the heliosphere whose outer boundary, the heliopause, is estimated to lie at $10^{2} \mathrm{AU}$ or beyond. So in the large the activity of the Sun dictates the conditions throughout the planetary system. Even the powerful galactic cosmic ray gas is swept away to some degree by the outward rush of magnetic field in the solar wind.

The radiative output of the Sun is modulated in important ways by the magnetic activity. It has been known for many decades that the electromagnetic radiation from the Sun, which has its peak intensity in yellow light, has a UV excess that extends into $\mathrm{X}$-rays as a consequence of the superheated transition layer and corona. Such radiation is the product of the suprathermal activity, and its intensity varies widely with the changing level of magnetic activity. The X-ray intensity at $10 \mathrm{keV}$ fluctuates by several decades over the magnetic cycle, with transient excursions up to a factor of $10^{10}$ at the time of a large flare (cf. Dennis 1985). The UV varies by several percent with the magnetic cycle.

The most remarkable aspect of the magnetic activity of the Sun is the variation $\Delta L_{\odot}$ of the total luminosity $L_{\odot}$ (the irradiance, as observed near the equatorial plane of the Sun) in proportion to the general level of activity. Direct observation by NIMBUS 7 shows a decrease by 2 parts in $10^{3}$ between 1979 and 1986 (Hoyt et al. 1992). The work of Radick et al. (1990) and Zhang et al. (1993) shows that there is a universal quantitative $\Delta L$ - activity relation for middle aged solar-type stars. Foukal \& Lean (1986) show that 
a large part of $\Delta L_{\odot}$ results from the competition between plages and sunspots, with the plages dominating so that the mean $L_{\odot}$ increases with the activity.

Now there are two different aspects to the problem of solar activity that should be clearly understood. First of all, although the magnetic activity has been studied for many decades, and has been described at length with an extensive vocabulary of technical terms, there is only a limited understanding of the physics of the various aspects of the activity. For instance, there is a variety of ideas on the form of the solar dynamo. The nature of the plage, the general fibril state of the magnetic field, the clustering of fibrils to form sunspots, and the basic reasons for $\Delta L_{\odot}$ are not clearly understood. That is to say, one cannot yet establish from the principles of physics why a star like the Sun is obliged to produce these phenomena. The heat sources that create the active X-ray corona and the quiet corona and the coronal hole have not been established for lack of the crucial observations of the small-scale behavior of the fibril magnetic field. The scientific program is seriously handicapped by the seeing limitations of the ground based telescope, and by the curious reluctance of NASA to fly a $1 \mathrm{~m}$ diffraction limited telescope either in orbit or with a balloon, to resolve the critical aspects of the activity at the Sun.

Second, there is enough known of the variation of the solar output, from IR to Xrays, to recognize the broad impact on the terrestrial atmosphere. The chemistry and general state of the upper atmosphere and the ionosphere are driven and controlled by the varying UV and X-rays. The ozone-UV problem is widely known and is an example of the complicated chemical interaction of anthropogenic pollutants in the variable UV irradiance. More recently the observational determination of a substantial $\Delta L_{\odot}$ indicates that the recorded century by century terrestrial forays into hot and cold climate in the northern temperate zone are evidently connected with the changing activity level and the corresponding change in the luminosity of the Sun. The human consequences of the climatic extremes are well recorded (Eddy 1976; 1977; Wigley \& Kelly 1990; FriisChristensen \& Lassen 1991) from the agricultural disasters in northern climes during the cold 15 th and 17 th centuries to the devastating dessication of southwestern United States in the warm 12th century. The scientific study of the effects should, therefore, be recognized as the central concern of the worldwide space science program.

It must be appreciated that the study of the variable activity and luminosity of the Sun extends across diverse fields of scientific inquiry. The oceanic and atmospheric sciences are directly involved, of course, at all levels through the upper atmosphere. Aeronomy and ionospheric physics and chemistry play a central role, which also involves magnetospheric convection and activity. The system is driven by changes across the solar spectrum, and in some degree by the solar wind and momentarily by transient bursts of energetic particles from the Sun. Therefore it poses a central problem for solar physics to quantify and explain the variability of the thermal, suprathermal and particle emissions from the Sun while the climatic consequences are studied by the geophysical and atmospheric sciences. The scientific community should be the first to recognize the many dimensions and the central priority of the solar variability - terrestrial environment problem, now that it is known to be a major player in the climate in both the upper and lower levels of the terrestrial atmosphere.

The remainder of this paper is directed to recent questions about the solar dynamo and the requirement of flux bundles of $10^{5}$ gauss in the overshoot layer below the convective zone in order to account for the location and orientation of the active bipolar magnetic regions at the surface of the Sun. The inquiry leads to a curious barometric effect in the buoyant rise of $\Omega$-loops of azimuthal field to the surface, and suggests that the increased luminosity may be an automatic consequence of the emergence of the active regions. 


\section{Origins of solar activity}

The magnetic field of the Sun, created and driven by the subsurface convection, is the basis for solar activity. It is a matter of conjecture whether the fibril state of the magnetic field observed at the surface implies a fibril state throughout the entire convective zone. (cf. Parker 1984; Zwaan 1985; see also Solanki 1993). The origin of the magnetic field appears to be an $\alpha \omega$ dynamo (Parker 1955, 1957). Helioseismology indicates that the principal shear (nonuniform rotation, $\partial \omega / \partial r$ ) is confined to a thin layer immediately below the base of the convective zone. Therefore it is presumed that the azimuthal field resides mainly in that layer. Dynamical studies of azimuthal field in the sheared subadiabatic temperature gradient below the convective zone (Spruit \& Van Ballegooijen 1982; Fisher et al. 1991; Moreno-Insertis et al. 1993) indicate that azimuthal fields up to about $10^{5}$ gauss can be stably confined, but that, when the field strength exceeds some such magnitude, it becomes unstable to the formation of $\Omega$-loops. It is presumably these $\Omega$-loops that emerge through the surface of the Sun to provide the observed bipolar magnetic active regions (Parker 1955). It is interesting to note that recent calculations by D'Silva \& Choudhuri $(1993)$ and Fan et al. $(1993,1994)$ on the dynamics of buoyant flux bundles show that $\Omega$ loops emerge through the surface at the proper latitude with the observed inclination to the east-west direction only if the emerging $\Omega$-loops are formed from fields of $0.5-1 \times 10^{5}$ gauss. Evidently, then, the conditions for the onset of buoyant instability in the shear layer determine the observed location and orientation of the bipolar magnetic regions at the surface.

Now the field intensity of $5 \times 10^{4}$ gauss does not follow from the available solar dynamo effects. To be precise, a nonuniform rotation of the order of $3 \times 10^{4} \mathrm{~cm} / \mathrm{sec}$ across a shear layer of thickness $h=3 \times 10^{9} \mathrm{~cm}$ below the convective zone yields

$$
\begin{aligned}
G & \equiv d v / d r \\
& \cong 10^{-5} / \mathrm{sec} .
\end{aligned}
$$

The rate of generation of azimuthal field $B_{\varphi}$ from a vertical poloidal field $B_{p}$ is

$$
\frac{\partial B_{\varphi}}{\partial t}=G B_{p}
$$

so that if $B_{\varphi}$ is to reach $5 \times 10^{4}$ gauss in 3 years it follows that $B_{p}=50$ gauss. The Maxwell stress $B_{\varphi} B_{p} / 4 \pi \cong 2 \times 10^{5}$ dynes $/ \mathrm{cm}^{2}$ would spread out the differential rotation from $h$ to $2 h$ in just half a year. Evidently, then, the $\alpha \omega$-dynamo of the Sun generates a mean azimuthal field of the order of a couple of kilogauss (Parker 1993), necessary to account for the $10^{23}$ Maxwells that appear in large bipolar magnetic regions at the surface (Gaizauskas et al. 1983). Some other effect concentrates the mean field into widely separated intense fibrils of $0.5-1 \times 10^{5}$ gauss.

We suggest that the concentration of the field is an automatic byproduct of the formation of $\Omega$-loops and their emergence through the visible surface of the Sun. It appears, too, that the formation of $\Omega$-loops may increase the vertical mixing of gas and entropy sufficiently as to account for the increase in the solar luminosity observed to accompany the magnetic activity of emerging $\Omega$-loops. The effect is a simple one. A buoyant azimuthal flux bundle gives rise to an $\Omega$-loop, driving a convective updraft all the way to the surface of the Sun. The essential point for concentrating the field is that the temperature within the rising $\Omega$-loop increases by the amount $\Delta T(z)$ above the ambient $T(z)$ at the same height $z$ by the amount

$$
\Delta T(z)=\int_{0}^{z} d z \Delta \frac{d T}{d z}
$$


where $\Delta d T / d z$ is the excess of the ambient temperature gradient $|d T / d z|$ over the nearly adiabatic temperature gradient of the rising fluid. The effect is, then, for the hotter gas within the field of the $\Omega$-loop to billow upward within the flux bundle, drawing gas up behind it from the feet of the $\Omega$-loop and hence from the azimuthal flux bundle in which it is formed. This partial evacuation of the azimuthal flux bundle shrinks the flux bundle and intensifies the field in the bundle. The limiting value of the field $B_{\max }$ is determined by the limiting value of the pressure reduction

$$
\Delta p(0) \cong p(0) \int_{0}^{z} \frac{d z}{\Lambda(z)} \frac{\Delta T(z)}{T(z}
$$

in the base of the $\Omega$-loop, where $\Lambda(z)$ is the pressure scale height $\mathrm{kT}(\mathrm{z}) / \mathrm{Mg}$.

To deduce this result formally, consider the momentum equation for a vertical flux bundle extending upward from the base $z=0$ of the convective zone. Then the fluid velocity $v$ along the bundle satisfies

$$
\frac{d v}{d t}=-\frac{1}{\rho} \frac{\partial p}{\partial z}-g
$$

where we may as well approximate the gravitational acceleration by a constant value of about $3 \times 10^{4} \mathrm{~cm} / \mathrm{sec}^{2}$. Multiply by $\mathrm{M} / \mathrm{kT}$ and integrate upward from $z=0$. The result can be written

$$
p(z)=p(0) \exp \left[-\int_{0}^{z} \frac{d s}{\Lambda(s)}-\int_{0}^{z} \frac{d s}{\Lambda(s)} \frac{1}{g} \frac{d v}{d t}\right] .
$$

The relation can be applied to the gas within the vertical flux bundle, where $d v / d t \neq 0$ and where the gas temperature is $T(z)+\Delta T(z)$, and it may be applied to the ambient gas outside $(d v / d t \cong 0)$ which has a temperature $T(z)$. Thus, for the ambient pressure outside,

$$
p(z)=p(0) \exp \left[-\int_{0}^{z} \frac{d s}{\Lambda(s)}\right] .
$$

Inside the flux bundle write $\Lambda(z)+\Delta \Lambda(z)$ for the scale height, with $\Delta \Lambda(z)=k \Delta T(z) /$ $M g$. Note, then, that $\Delta \Lambda / \Lambda$ and $g^{-1} d v / d t$ are both small compared to one, so that to first order, the pressure $p_{i}(z)$ inside the bundle is

$$
p_{i}(z) \cong \frac{p_{i}(0)}{p(0)} p(z)\left[1+\int_{0}^{z} d z \frac{\Delta \Lambda}{\Lambda^{2}}-\frac{1}{g} \int_{0}^{z} \frac{d t}{\Lambda} \frac{d v}{d t}\right] .
$$

This equation may now be used to compute

$$
\Delta p(z)=p(z)-p_{i}(z)
$$

in terms of $\Delta p(0)$, yielding

$$
\frac{\Delta p(0)}{p(0)}=\frac{\Delta p(z)}{p(z)}+\int_{0}^{z} \frac{d z \Delta \Lambda(z)}{\Lambda^{2}(z)}-\frac{1}{g} \int_{0}^{z} \frac{d z}{\Lambda(z)} \frac{d v}{d t}
$$

upon neglecting terms second order in $\Delta p / p$.

The condition for local transverse equilibrium of the slender flux bundle is $B^{2}(z) / 8 \pi=$ $\Delta p(z)$, of course, so that

$$
\frac{B^{2}(0)}{8 \pi}=p(0)\left\{\frac{B^{2}(z)}{8 \pi p(z)}+\int_{0}^{z} \frac{d z \Delta \Lambda(z)}{\Lambda(z)^{2}}-\frac{1}{g} \int_{0}^{z} \frac{d z d v / d t}{\Lambda(z)}\right\} .
$$

In the simplest case $d v / d t=0$ and the field at the apex $z=\lambda$ of the $\Omega$-loop expands so much that the first term in braces can be neglected. There remains the basic barometric 
term

$$
\frac{B^{2}(0)}{8 \pi}=p(0) \int_{0}^{\lambda} \frac{d z}{\Lambda(z)} \frac{\Delta T}{T(z)} .
$$

A standard convective zone model (Spruit 1974) gives $\Lambda(0)=7 \times 10^{9} \mathrm{~cm}$ at the bottom of the convective zone $(z=0)$ where $p(0)=6 \times 10^{13}$ dynes $/ \mathrm{cm}^{2}$ and $T(0)=2 \times 10^{6} \mathrm{~K}$. At the midlevel $\left(z=10^{10} \mathrm{~cm}\right) p=6 \times 10^{12}$ dynes $/ \mathrm{cm}^{2}, T \cong 10^{6} \mathrm{~K}$, and $\Lambda \cong 4 \times 10^{9} \mathrm{~cm}$, with $\Delta T \cong 1 \mathrm{~K}$. At the three quarter level $\left(z=1.5 \times 10^{10} \mathrm{~cm}\right) p=10^{12}$ dynes $/ \mathrm{cm}^{2} T=4 \times 10^{5}$, and $\Delta T \cong 6 \mathrm{~K}$. We find at the three quarter level that the integral of $\Delta \Lambda / \Lambda^{2}$ reaches a value of $10^{-5}$ and increases rapidly upward from there with rapidly increasing $\Delta T$ and decreasing $\Lambda$. Thus the limiting pressure reduction is $\Delta p(0) \cong 10^{-5} p(0) \cong 6 \times 10^{8}$ dynes $/ \mathrm{cm}^{2}$, equivalent to $B(0) \cong 1.2 \times 10^{5}$ gauss. So the $\Omega$-pumping is capable of limiting fields $B_{\max }$ of the required order of $10^{5}$ gauss.

One imagines that the process begins with azimuthal fields of a few kilogauss at middle latitudes where the nonuniform rotation $d \omega / d r$ is not very strong. The convective overshoot may initiate an $\Omega$-loop in spite of the stability of the mean field. The $\Omega-$ pumping begins, eventually concentrating many, if not all, of the azimuthal flux bundles into intense separated magnetic fibrils of $0.5 \times 10^{5}$ gauss or more.

The process is certainly more complicated than this simple idealized example. For instance the term $B^{2}(s) / 8 \pi p(s)$ on the right hand side of equation (8) can only increase the effect, perhaps substantially. On the other hand, the third term on the right hand side represents inertial effects and reduces the effect where $d v / d t$ is positive (upward). In fact $d v / d t$ may have either sign, but there is no reason to think that the effect is large either way. For instance, the upward motion $v$ of an $\Omega$-loop is presumably of the order of $2 \times 10^{4} \mathrm{~cm} / \mathrm{sec}$, covering the thickness $2 \times 10^{10} \mathrm{~cm}$ of the convective zone in a time $\tau=10^{6} \mathrm{sec}$. The characteristic mean upward acceleration is, then, $2 v / \tau=4 \times 10^{-2}$ $\mathrm{cm} / \mathrm{sec}^{2}$. Hence $2 v / g \tau=1.3 \times 10^{-6}$ and is integrated over only a few scale heights, so that the integral may approach $10^{-5}$. Remembering that the vertical motion decelerates to zero at the surface, where there are many scale heights, the net effect of the inertia is not only small but may actually contribute to the net effect as the $\Omega$-loop approaches the surface.

\section{Summary}

The emergence of $\Omega$-loops from the azimuthal field to provide the magnetic activity at the surface of the Sun poses a problem as to what effect can convert the mean kilogauss azimuthal field in the overshoot-shear layer into a fibril field with individual intensities of the order of $10^{5}$ gauss. It looks as though it may be the emergence of the $\Omega$-loops themselves that is responsible, through the $\Omega$-pumping effect. Moreno-Insertis $\&$ Schüssler (1990) have pointed out an intensification of the vertical legs of $\Omega$-loops in their numerical experiments on the dynamics of $\Omega$-loop, resembling the $\Omega$-pumping described above.

As a final point, the vigorous buoyant rise of $\Omega$-loops, pushing all the way through from the bottom to the top of the convective zone, may be expected to enhance the total upward convective heat transport by substantial amounts. It may be an important factor in producing the enhanced solar luminosity during the years of high activity and clearly needs quantitative consideration.

Acknowledgments. This work was supported in part by the National Aeronautics and Space Administration under NASA grant NAGW-2122. 


\section{REFERENCES}

DENNIS, B.R. 1985 Solar hard X-ray bursts. Solar Phys. 100, 465-490.

D'Silva, S. \& ChoudhuRI, A.R. 1993 A theoretical model for tilts of bipolar magnetic regions. Astron. Astrophys. 272, 621-633.

EDDY, J.A. 1976 The Maunder Minimum. Science 192, 1189-1202.

EDDY, J.A. 1977 Historical evidence for the existence of the solar cycle. In Solar Output and its Variation (ed. O.R. White). pp. 51-77. Colorado Association University Press.

Fan, Y., Fisher, G.H. \& DeLucA, E.E. 1993 The origin of morphological asymmetries in bipolar active regions. Astrophys. J. 405, 390-401.

Fan, Y., Fisher, G.H. \& McClymont, A.N. 1994 Astrophys. J., submitted.

Fisher, G.H., McClymont, A.N. \& Chou, D.Y. 1991 The strerching of magnetic flux tubes in the convective overshoot region. Astrophys. J. 374, 766-772.

FoukAL, P. \& LEAN, J. 1986 The influence of faculae on total solar irradiance and luminosity. Astrophys. J. 302, 826-835.

Frils-Christenson, E. \& LAsSen, K. 1991 Length of the solar cycle: An indicator of solar activity closely associated with climate. Science 254, 698-700.

Gaizauskas, V., Harvey, K.L., Harvey, J.W. \& ZwaAn, C. 1983 Large-scale patterns formed by solar active regions during the ascending phase of cycle 21 . Astrophys. $J \mathbf{2 6 5}$, $1056-1065$.

Hoyt, D.V., Kyle, H.L., Hickey, J.R. \& Maschnoff, R.H. 1992 The Nimbus 7 total irradiance: A new algorithm for its deviation. J. Geophys. Res. 97, 51-63.

Moreno-Insertis, F., Schüssler M., \& Feriz-Mas, A. 1984 Private communication.

Moreno-Insertis, F., Schüssler, M. \& Feriz-Mas, A. 1993 Astron. Astrophys., submitted.

PARKER, E.N. 1955 The formation of sunspots from the solar toroidal field. Astrophys. J. 121, 491-507.

Parker, E.N. 1984 Stellar fibril systems: I. Reduced energy state. Astrophys. J. 283, 343-348.

PARKER, E.N. 1993 A solar dynamo surface wave at the interface between convection and nonuniform rotation. Astrophys. J. 408, 707-719.

Radick, R.R., LockWood, G.W. \& Baliunas, S.L. 1990 Solar activity and brightness variations: A glimpse at the Sun's history. Science 24, 39-44.

SolankI, S.K. 1993 Small-scale solar magnetic fields: An overview. Space Sci. Rev. 63, 1-186.

Spruit, H.C. 1974 A model of the solar convective zone. Solar Phys. 34, 277-290.

Spruit, H.C. \& van Ballegooluen, A.A. 1982 Stability of toroidal flux tubes in stars. Astron. Astrophys. 113, 350.

Wigley, T.M.L. \& KeLly, P.M. 1990 Holocene climatic change, ${ }^{14} \mathrm{C}$ wiggles and variations in solar irradiance. Phil. Trans. Roy. Soc. London A330, pp. 547-560.

Zhang, Q., Soon, W.H., Baliunas, S.L., Lockwood, G.W., SkifF, B.A. \& Radick, R.R. 1993 Possible method for determining brightness variations of the Sun in past centuries from solar type stars Astrophys. J. Letters, submitted.

ZWAAN, C. 1985 The emergence of magnetic flux. Solar Phys. 100, 397-414. 